\title{
Stereotactic Body Radiation Therapy (SBRT) for clinically localized prostate cancer: the Georgetown University experience
}

Leonard N Chen ${ }^{1 \dagger}$, Simeng Suy ${ }^{1 \dagger}$, Sunghae Uhm¹, Eric K Oermann ${ }^{1}$, Andrew W Ju', Viola Chen ${ }^{1}$,

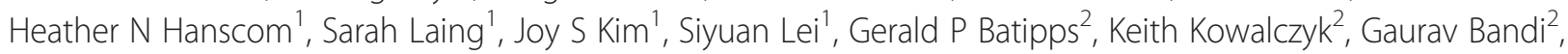
John Pahira ${ }^{2}$, Kevin G McGeagh², Brian T Collins ${ }^{1}$, Pranay Krishnan ${ }^{3}$, Nancy A Dawson ${ }^{4}$, Kathryn L Taylor ${ }^{4}$, Anatoly Dritschilo ${ }^{1}$, John H Lynch ${ }^{2}$ and Sean P Collins ${ }^{1 *}$

\begin{abstract}
Background: Stereotactic body radiation therapy (SBRT) delivers fewer high-dose fractions of radiation which may be radiobiologically favorable to conventional low-dose fractions commonly used for prostate cancer radiotherapy. We report our early experience using SBRT for localized prostate cancer.

Methods: Patients treated with SBRT from June 2008 to May 2010 at Georgetown University Hospital for localized prostate carcinoma, with or without the use of androgen deprivation therapy (ADT), were included in this retrospective review of data that was prospectively collected in an institutional database. Treatment was delivered using the Cyberknife ${ }^{\circledR}$ with doses of 35 Gy or 36.25 Gy in 5 fractions. Biochemical control was assessed using the Phoenix definition. Toxicities were recorded and scored using the CTCAE v.3. Quality of life was assessed before and after treatment using the Short Form-12 Health Survey (SF-12), the American Urological Association Symptom Score (AUA) and Sexual Health Inventory for Men (SHIM) questionnaires. Late urinary symptom flare was defined as an AUA score $\geq 15$ with an increase of $\geq 5$ points above baseline six months after the completion of SBRT.
\end{abstract}

Results: One hundred patients (37 low-, 55 intermediate- and 8 high-risk according to the D'Amico classification) at a median age of 69 years (range, 48-90 years) received SBRT, with 11 patients receiving ADT. The median pretreatment prostate-specific antigen (PSA) was $6.2 \mathrm{ng} / \mathrm{ml}$ (range, $1.9-31.6 \mathrm{ng} / \mathrm{ml}$ ) and the median follow-up was 2.3 years (range, 1.4-3.5 years). At 2 years, median PSA decreased to $0.49 \mathrm{ng} / \mathrm{ml}$ (range, $0.1-1.9 \mathrm{ng} / \mathrm{ml}$ ). Benign PSA bounce occurred in $31 \%$ of patients. There was one biochemical failure in a high-risk patient, yielding a two-year actuarial biochemical relapse free survival of $99 \%$. The 2-year actuarial incidence rates of GI and GU toxicity $\geq$ grade 2 were $1 \%$ and $31 \%$, respectively. A median baseline AUA symptom score of 8 significantly increased to 11 at 1 month $(p=0.001)$, however returned to baseline at 3 months $(p=0.60)$. Twenty one percent of patients experienced a late transient urinary symptom flare in the first two years following treatment. Of patients who were sexually potent prior to treatment, $79 \%$ maintained potency at 2 years post-treatment.

(Continued on next page)

\footnotetext{
* Correspondence: spc9@gunet.georgetown.edu

${ }^{\dagger}$ Equal contributors

'Department of Radiation Medicine, Georgetown University Hospital, Washington, DC 20007, USA

Full list of author information is available at the end of the article
} 
(Continued from previous page)

Conclusions: SBRT for clinically localized prostate cancer was well tolerated, with an early biochemical response similar to other radiation therapy treatments. Benign PSA bounces were common. Late Gl and GU toxicity rates were comparable to conventionally fractionated radiation therapy and brachytherapy. Late urinary symptom flares were observed but the majority resolved with conservative management. A high percentage of men who were potent prior to treatment remained potent two years following treatment.

Keywords: Prostate cancer, SBRT, CyberKnife, SHIM, AUA, SF-12, Quality of life, Common Toxicity Criteria (CTC), Benign PSA bounce, Urinary symptom flare

\section{Background}

For men with localized prostate cancer, the typical treatment with dose-escalated external beam radiation therapy (EBRT) involves fractionated radiation therapy using daily doses of 1.8-2.0 Gy for eight to nine weeks. Considering logistics and life responsibilities, such prolonged treatment courses present hardship for many patients. In addition, clinical data suggest that hypofractionated radiation therapy may be radiobiologically favorable to smaller fraction sizes in prostate cancer radiotherapy due to a potentially greater sensitivity of prostate cancer to larger daily radiation fractions [1]. Early data from trials of limited hypofractionation (fraction sizes from 2.5 to $3.5 \mathrm{~Gy}$ ) revealed that such regimens are effective without undue toxicity [2]. Stereotactic body radiation therapy (SBRT) uses even larger daily fractions of radiation to take further advantage of this postulated radiobiological advantage. Early investigations of SBRT were performed with radiation delivery systems that did not allow continuous tracking of the prostate's location with intrafractional adjustment of beam targeting if motion was detected [3]. Initially, the goal was to maintain a similar level of local control while sparing normal tissue using fairly low doses (33.5 Gy in five fractions). This relatively low biologically equivalent dose, perhaps in combination with geographic misses due to inadequate margins, led to relatively poor biochemical control [3].

CyberKnife $^{\circledR}$ (Accuray Incorporated, Sunnyvale, CA) delivers hundreds of individualized circular beams with a targeting error of less than $1 \mathrm{~mm}$ allowing the safe delivery of highly conformal treatment plans with steep dose gradients [4]. Unlike standard image-guided radiation therapy (IGRT), the CyberKnife system incorporates a real-time tracking system that provides updated prostate position information to the robot to correct the targeting of the therapeutic beam during treatment [5]. This feature allows for a reduction in the planning target volume (PTV) and, therefore, better limits the dose to surrounding critical organs. Such technology has enabled other institutions to administer SBRT to the prostate (doses of 35-36.25 Gy) with excellent biochemical disease-free survival yet with toxicities similar to conventional treatments [6-8]. Here we present our early institutional experience with SBRT for clinically localized prostate cancer.

\section{Methods}

\section{Patient selection}

Patients eligible for study inclusion had histologicallyconfirmed adenocarcinoma of the prostate treated per our institutional protocol. Exclusion criteria included clinical stage T3, involved lymph nodes or distant metastases on imaging and/or prior pelvic radiotherapy. Institutional IRB approval was obtained for retrospective review of data that was prospectively collected in our institutional database.

\section{SBRT treatment planning and delivery}

Four gold fiducials were placed into the prostate. Seven days after fiducial placement, patients underwent MR imaging followed shortly thereafter by a thin-cut CT scan. Fused CT and MR images were used for treatment planning. The clinical target volume (CTV) included the prostate and the proximal seminal vesicles (to the point where the seminal vesicles separate). The PTV equaled the CTV expanded $3 \mathrm{~mm}$ posteriorly and $5 \mathrm{~mm}$ in all other dimensions. The prescription dose was $35-36.25$ Gy to the PTV delivered in five fractions of 7-7.25 Gy corresponds to a tumor EQD2 of approximately 85-90 Gy assuming an $\alpha / \beta$ ratio of 1.5. In general, older patients with poor baseline urinary function were treated with $35 \mathrm{~Gy}$.

Treatment plans were composed of hundreds of pencil beams using one to two circular collimator to generate highly conformal plans (mean new conformity index of 1.28 [range, 1.12-1.59]). Plans were inhomogeneous by design (mean homogeneity index of 1.29 [range, 1.23-1.42]) to minimize dose to adjacent critical structures (Figure 1a). However, the prescription isodose line was limited to $\geq$ $75 \%$ to restrict the maximum prostatic urethra dose to $133 \%$ of the prescription dose. The rectum, bladder, testes, penile bulb and membranous urethra were contoured and evaluated with dose-volume histogram analysis during treatment planning using Multiplan (Accuray Inc., Sunnyvale, CA) inverse treatment planning. A typical dosevolume histogram is shown in Figure $1 \mathrm{~b}$ and critical structure dose constraints are shown in Table 1. To minimize 

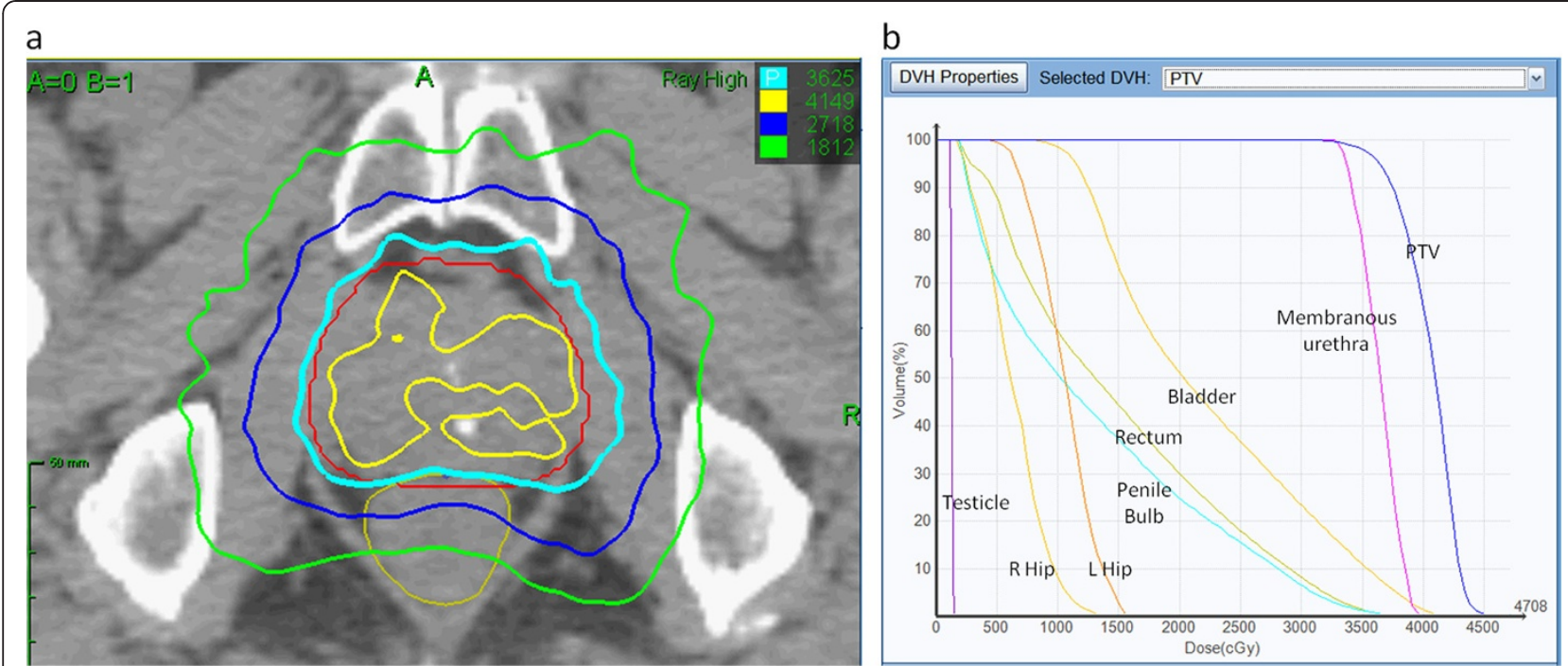

Figure 1 (a) Treatment planning axial computed tomography images demonstrating the prostate (red line) and rectum (brown line). Isodose lines shown as follows: 115\% of the prescription dose, yellow line; 100\% of the prescription dose, light blue line: $75 \%$ of the prescription dose, dark blue line; and $50 \%$ of the prescription dose, green line. (b) A typical dose-volume histogram for CyberKnife treatment of a prostate cancer patient.

the risk of local recurrence, no attempt was made to limit the dose to the prostatic urethra or neurovascular bundles $[9,10]$. Radiation was delivered every other day to a mean prescription isodose line of $77 \%$ (range, $75-81 \%$ ) in 5 approximately 1 hour long treatments, with a mean treatment duration of 10.6 days (range, 5-16 days). On average, 247 beams were employed (range, 199-289 beams) to treat the mean prescription volume of $135 \mathrm{cc}$ (range, 61-258 cc) with mean percent target coverage of 95.20\% (range, 93.65-96.95\%). Target position was verified

Table 1 Dose targets and constraints for treatment planning

\begin{tabular}{ll}
\hline \multicolumn{2}{c}{36.25 Gy plan constraints } \\
\hline Global Max Dose & $48.33 \mathrm{~Gy}$ \\
PTV & $\mathrm{V}(36.25 \mathrm{~Gy}) \geq 95 \%$ \\
Rectum & $\mathrm{V}(36 \mathrm{~Gy})<1 \mathrm{cc}$ \\
& $\mathrm{V}(100 \%)<5 \%$ \\
& $\mathrm{~V}(90 \%)<10 \%$ \\
& $\mathrm{~V}(80 \%)<20 \%$ \\
& $\mathrm{~V}(75 \%)<25 \%$ \\
& $\mathrm{~V}(50 \%)<50 \%$ \\
Bladder & $\mathrm{V}(37 \mathrm{~Gy})<5 \mathrm{cc}$ \\
& $\mathrm{V}(100 \%)<10 \%$ \\
Penile Bulb & $\mathrm{V}(50 \%)<40 \%$ \\
Membranous urethra & $\mathrm{V}(29.5 \mathrm{~Gy})<50 \%$ \\
Sigmoid colon & $\mathrm{V}(37 \mathrm{~Gy})<50 \%$ \\
Testicles & $\mathrm{V}(30 \mathrm{~Gy})<1 \mathrm{cc}$ \\
\hline
\end{tabular}

every 30-60 seconds during treatment using paired, orthogonal $\mathrm{kV}$ images (total imaging effective dose equals $17.5 \mathrm{mSv})$.

\section{Follow-up and statistical analysis}

Prostate-specific antigen (PSA) and total testosterone levels were obtained before treatment, one month after the completion of SBRT, and during routine follow-up visits every 3 months for the first year and every six months for the second year of follow-up. Alphaantagonist, antidiarrheal and phosphodiesterase type 5 (PDE5) inhibitor utilization was documented at each visit.

Toxicity was prospectively documented at follow-up visits using the National Cancer Institute (NCI) Common Toxicity Criteria (CTC) version 3.0. Acute toxicity was defined as experiencing toxicity during or within 6 months of radiation therapy. Late toxicity was defined as occurring at least 6 months after delivery of radiation therapy. Transient and chronic late toxicities were included. The genitourinary toxicities analyzed were hematuria, dysuria, incontinence, urinary urgency/frequency and retention. The gastrointestinal toxicities analyzed were bowel frequency/urgency, proctitis and rectal bleeding. In general, Grade 1 toxicity represents minimal side effect not requiring medications for symptom control. Pre-treatment symptoms were counted as Grade 1 toxicity if they increased in severity. Grade 2 toxicity indicates symptoms requiring new medication (i.e. alphaantagonist or antidiarrheal) or increase in dose of previously prescribed medication. Grade 3 indicates complications requiring minor surgical intervention (i.e., 
transurethral resection or laser coagulation). At each follow-up visit, toxicity events were scored independently for each of the different toxicity types and the highest GU and GI toxicity was determined for each patient.

Quality of life (QOL) was assessed before and after treatment using the Short Form-12 Health Survey (SF-12), the American Urological Association Symptom Score (AUA) and Sexual Health Inventory for Men (SHIM) questionnaires. The Medical Outcomes Study Short Form-12 Health Survey (SF-12) [11], which contains two subscales, the Mental Component Summary (MCS) and the Physical Component Summary (PCS), with higher values indicating better quality of life. AUA scores range from 0-35 with higher values representing worsening urinary symptoms [12]. SHIM scores range from $0-25$ with lower values representing worsening sexual symptoms [13].

Student's $t$-test and chi-square analysis were used to assess differences in ongoing PSA, testosterone and quality of life scores (SF-12, AUA and SHIM) in comparison to baseline. Sample medians and ranges were used to describe continuous variables including PSA and testosterone. Based upon published results, a benign PSA bounce was defined as a PSA rise of $0.2 \mathrm{ng} / \mathrm{mL}$ or more above its previous nadir with a subsequent decline to that nadir or lower [14]. Actuarial likelihood estimates for late toxicities were determined using the Kaplan-Meier method. The highest GU or GI toxicity available for each patient was evaluated for the actuarial analysis. SF-12 analysis with norm-based scores $($ Mean $=50$, standard deviation $=10)$ [15] was performed using IBM SPSS Statistics 20. The minimally important difference (MID) in AUA score was defined as a change of one-half standard deviation (SD) from the baseline [16]. As previously reported, late urinary symptom flare was defined as an increase of $\geq 5$ points above baseline with a degree of severity in the moderate to severe range (AUA score $\geq 15$ ) [17]. The flare was considered resolved when either the AUA score dropped to $<15$ or the score returned to $<5$ points above the patient's pretreatment baseline. Erectile dysfunction (ED) was categorized into five categories of severity based on the patient's SHIM score: no ED (22-25), mild ED (17-21), mild to moderate (12-16), moderate (8-11) and severe (1-7) [18]. Patients were considered potent if they scored $\geq 10$ on the SHIM [18]. To limit the effect of attrition bias, statistical analysis was limited to time points in which $\geq 80 \%$ of the patient data were available.

\section{Results}

From June 2008 to May 2010, 100 prostate cancer patients were treated per our institutional SBRT monotherapy protocol (Table 2). They were ethnically diverse with $57 \%$ being of Caucasian ancestry and a median age of 69 years (range, 48-90 years). By D’Amico classification,
Table 2 Patient characteristics and treatment

\begin{tabular}{|c|c|}
\hline Age (yrs) & Percent Patients $(n=100)$ \\
\hline$<60$ & 8 \\
\hline $60-69$ & 45 \\
\hline $70-79$ & 43 \\
\hline$\geq 80$ & 4 \\
\hline \multicolumn{2}{|l|}{ Race } \\
\hline White & 56 \\
\hline Black & 37 \\
\hline Hispanic & 5 \\
\hline Asian & 2 \\
\hline \multicolumn{2}{|l|}{ Pre-Tx PSA (ng/ml) } \\
\hline$\leq 10$ & 87 \\
\hline$>10$ and $\leq 20$ & 12 \\
\hline$>20$ & 1 \\
\hline \multicolumn{2}{|l|}{ T Stage } \\
\hline $\mathrm{T} 1 \mathrm{~b}$ & 1 \\
\hline T1c & 75 \\
\hline T2a & 12 \\
\hline $\mathrm{T} 2 \mathrm{~b}$ & 8 \\
\hline $\mathrm{T} 2 \mathrm{C}$ & 4 \\
\hline \multicolumn{2}{|l|}{ Gleason Score } \\
\hline $2+3=5$ & 2 \\
\hline $3+2=5$ & 1 \\
\hline $3+3=6$ & 42 \\
\hline $3+4=7$ & 40 \\
\hline $4+3=7$ & 10 \\
\hline $3+5=8$ & 1 \\
\hline $4+4=8$ & 4 \\
\hline \multicolumn{2}{|l|}{ Risk Groups } \\
\hline Low Risk & 37 \\
\hline Intermediate Risk & 55 \\
\hline High Risk & 8 \\
\hline \multicolumn{2}{|l|}{ Hormone Treatment } \\
\hline Yes & 11 \\
\hline No & 89 \\
\hline \multicolumn{2}{|l|}{ Dose (Gy) } \\
\hline 35.00 & 15 \\
\hline 36.25 & 85 \\
\hline
\end{tabular}

37 patients were low-, 55 intermediate-, and 8 high-risk. Eleven patients also received androgen deprivation therapy (ADT). Most received short term ADT (three to six months), while two high risk patients received long term ADT (two to three years). Eighty-five percent of the patients were treated with 36.25 Gy in five 7.25 Gy fractions. 
The median follow-up was 2.3 years (range, 1.4-3.5 years). At two years post-treatment, the median pretreatment PSA of $6.2 \mathrm{ng} / \mathrm{ml}$ (range, 1.9-31.6 ng/ml) declined to a median of $0.49 \mathrm{ng} / \mathrm{ml}$ (range, $0.1-1.9 \mathrm{ng} / \mathrm{ml}$ ) (Figure 2a). Benign PSA bounces occurred in $31 \%$ of patients with a median PSA bounce of $0.5 \mathrm{ng} / \mathrm{ml}$ (range, $0.2-2.2 \mathrm{ng} / \mathrm{ml}$ ) and the median time following treatment to the PSA bounce was 15 months (range, 3-21 months). There was one biochemical failure, occurring in a highrisk patient. Prostate biopsy confirmed local recurrence and ADT was initiated. The overall two-year actuarial biochemical relapse free survival was $99 \%$.

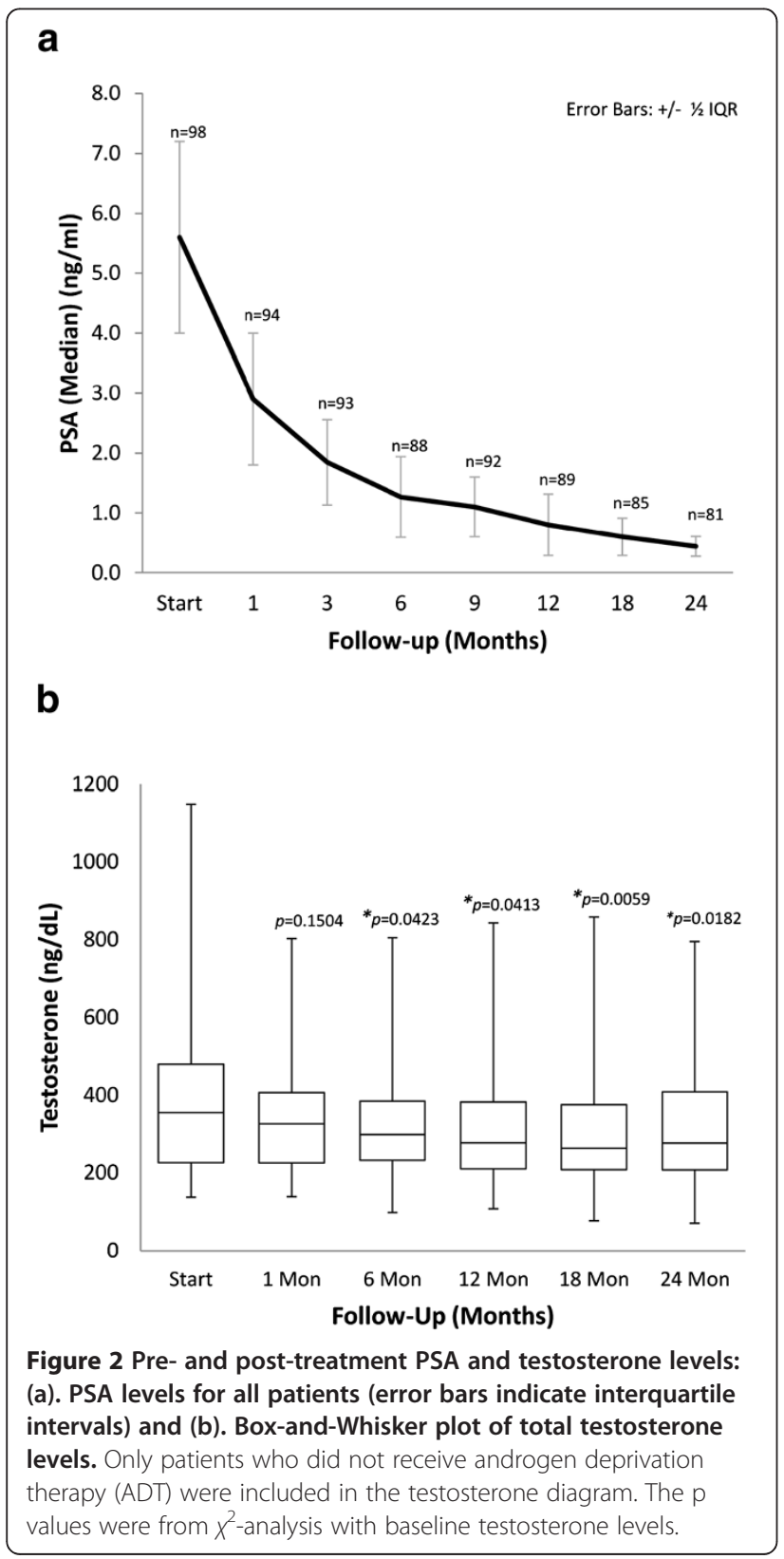

Pre-treatment total serum testosterone levels ranged from $4.75 \mathrm{nmol} / \mathrm{L}$ to $39.84 \mathrm{nmol} / \mathrm{L}$ with a median value of $12.29 \mathrm{nmol} / \mathrm{L}$. Twenty-nine percent of patients were hypogonadal (total serum testosterone level below $8 \mathrm{nmol} / \mathrm{L}$ ) prior to SBRT. At two years the median serum testosterone value of $9.78 \mathrm{nmol} / \mathrm{L}$ (range, $2.46-27.60 \mathrm{nmol} / \mathrm{L}$ ) was significantly lower than the pre-treatment value $(p<0.02$, Figure $2 \mathrm{~b})$. The median absolute reduction was small (2.5 $\mathrm{nmol} / \mathrm{L}$ ) and the median percent reduction was $20.5 \%$. The pre-treatment and 2-year biochemical hypogonadism

Table 3 Prevalence of CTC graded gastrointestinal (GI) and genitourinary (GU) toxicities at each follow-up

\begin{tabular}{|c|c|c|c|c|c|c|c|c|}
\hline \multicolumn{2}{|l|}{ Follow-up (months) } & \multirow{2}{*}{$\begin{array}{c}1 \\
\%\end{array}$} & \multirow{2}{*}{$\begin{array}{c}3 \\
\%\end{array}$} & \multirow{2}{*}{$\begin{array}{c}6 \\
\%\end{array}$} & \multirow{2}{*}{$\begin{array}{l}9 \\
\%\end{array}$} & \multirow{2}{*}{$\begin{array}{l}12 \\
\%\end{array}$} & \multirow{2}{*}{$\begin{array}{l}18 \\
\%\end{array}$} & \multirow{2}{*}{$\begin{array}{l}24 \\
\%\end{array}$} \\
\hline Toxicity & Grade & & & & & & & \\
\hline \multirow[t]{3}{*}{ Bowel Frequency/Urgency } & 0 & 72 & 83 & 78 & 81 & 91 & 95 & 93 \\
\hline & 1 & 23 & 16 & 20 & 18 & 8 & 5 & 7 \\
\hline & 2 & 5 & 1 & 1 & 1 & 1 & 0 & 0 \\
\hline \multirow[t]{3}{*}{ Proctitis } & 0 & 86 & 97 & 95 & 95 & 99 & 99 & 100 \\
\hline & 1 & 14 & 3 & 5 & 5 & 1 & 1 & 0 \\
\hline & 2 & 0 & 0 & 0 & 0 & 0 & 0 & 0 \\
\hline \multirow[t]{3}{*}{ Rectal Bleeding } & 0 & 94 & 98 & 96 & 95 & 98 & 93 & 96 \\
\hline & 1 & 6 & 2 & 4 & 5 & 2 & 7 & 4 \\
\hline & 2 & 0 & 0 & 0 & 0 & 0 & 0 & 0 \\
\hline \multirow[t]{3}{*}{ Highest Gl } & 0 & 60 & 79 & 71 & 73 & 87 & 89 & 89 \\
\hline & 1 & 35 & 20 & 28 & 26 & 12 & 11 & 11 \\
\hline & 2 & 5 & 1 & 1 & 1 & 1 & 0 & 0 \\
\hline \multirow[t]{4}{*}{ Hematuria } & 0 & 96 & 97 & 99 & 96 & 97 & 98 & 94 \\
\hline & 1 & 4 & 2 & 0 & 3 & 2 & 1 & 4 \\
\hline & 2 & 0 & 1 & 0 & 0 & 0 & 0 & 0 \\
\hline & 3 & 0 & 0 & 1 & 1 & 1 & 1 & 1 \\
\hline \multirow[t]{3}{*}{ Dysuria } & 0 & 71 & 91 & 97 & 88 & 93 & 96 & 93 \\
\hline & 1 & 29 & 9 & 3 & 13 & 7 & 4 & 7 \\
\hline & 2 & 0 & 0 & 0 & 0 & 0 & 0 & 0 \\
\hline \multirow[t]{3}{*}{ Incontinence } & 0 & 85 & 86 & 86 & 84 & 89 & 89 & 88 \\
\hline & 1 & 13 & 14 & 14 & 16 & 11 & 10 & 12 \\
\hline & 2 & 2 & 0 & 0 & 0 & 0 & 1 & 0 \\
\hline \multirow[t]{3}{*}{ Urinary Frequency/Urgency } & 0 & 81 & 90 & 90 & 93 & 93 & 90 & 88 \\
\hline & 1 & 19 & 10 & 9 & 7 & 6 & 8 & 11 \\
\hline & 2 & 0 & 0 & 1 & 0 & 1 & 2 & 1 \\
\hline \multirow[t]{3}{*}{ Retention } & 0 & 55 & 64 & 63 & 70 & 75 & 70 & 76 \\
\hline & 1 & 10 & 11 & 14 & 11 & 9 & 11 & 9 \\
\hline & 2 & 35 & 24 & 23 & 19 & 16 & 19 & 16 \\
\hline \multirow[t]{4}{*}{ Highest GU } & 0 & 28 & 52 & 53 & 54 & 59 & 53 & 57 \\
\hline & 1 & 36 & 23 & 23 & 26 & 23 & 26 & 26 \\
\hline & 2 & 35 & 25 & 23 & 19 & 17 & 20 & 17 \\
\hline & 3 & 0 & 0 & 1 & 1 & 1 & 1 & 1 \\
\hline
\end{tabular}


rates were not statistically significantly different (data not shown).

The prevalence of $\mathrm{GU}$ and GI toxicities following treatment is shown in Table 3. The prevalence of single symptoms as well as the highest GI and GU toxicity per patient are depicted independently for each follow-up visit. Acute urinary grade 2 toxicities requiring alphaantagonists occurred in $35 \%$ of patients (Table 3). Acute bowel frequency and/or spasms requiring anti-diarrheals were uncommon (5\%). The 2-year actuarial incidence rates of late GI and GU toxicity $\geq$ grade 2 were $1 \%$ and $31 \%$, respectively. Actuarial incidence rates of late grade 2 and $3 \mathrm{GU}$ toxicities are demonstrated in Figure 3. Grade 3 toxicities rates were low with one case of hematuria requiring transurethral resection of the prostate (TURP). The patient's prostatic volume was 85 grams and his pretreatment AUA score was 18. At two year post-treatment, the patients' perceptions of their physical health (Figure 4a) and mental health (Figure 4b) were not statistically different from baseline $(p=0.76$ and 0.90 , respectively).

The majority of patients had mild to moderate lower urinary tract symptoms prior to treatment with a mean baseline AUA of 8 (range, 0-24) (Table 4). At one month post-treatment the mean AUA symptom score increased to 11 , returning to baseline at 3 months $(p=0.60$, Figure $5 \mathrm{a})$. This increase was statistically significant $(p=0.001)$ but of borderline clinical significance $(\mathrm{MID}=3.22)$. Alphaantagonist utilization peaked at one month post-treatment at $65 \%$ patient utilization then slowly decreased to near baseline, with $40 \%$ of patients reporting use at two years (Figure 5b). Transient late urinary symptom flare ( $\geq 6$ months after completing treatment) occurred in $21 \%$ of the patients (Figure 5c). The median flare magnitude was 9 (range, 5-22), the median time to flare was 9 months (range, 6-21 months), and the median duration of flare

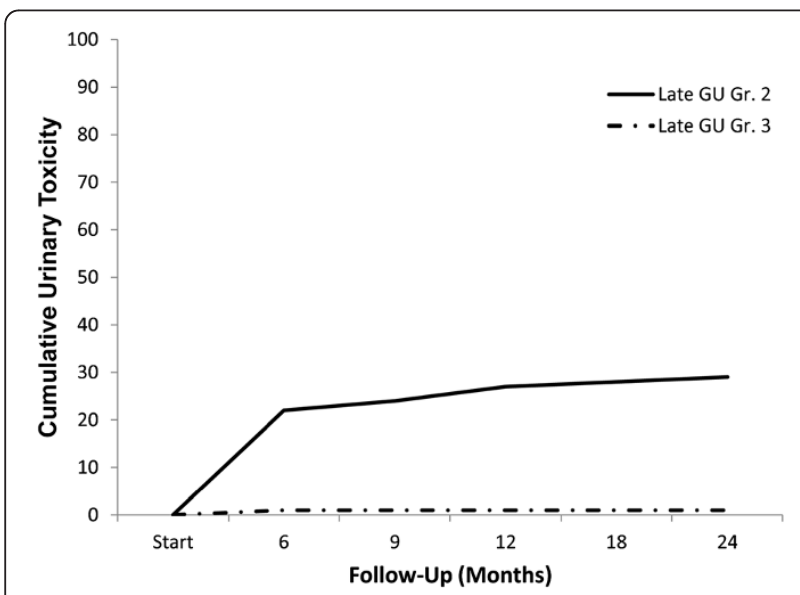

Figure 3 Cumulative late urinary toxicity (grades 2 and 3).

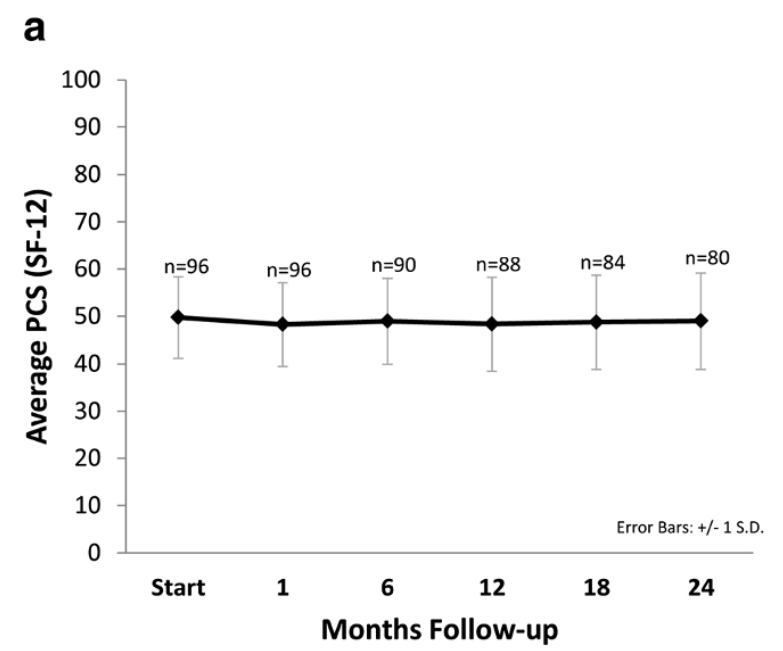

b

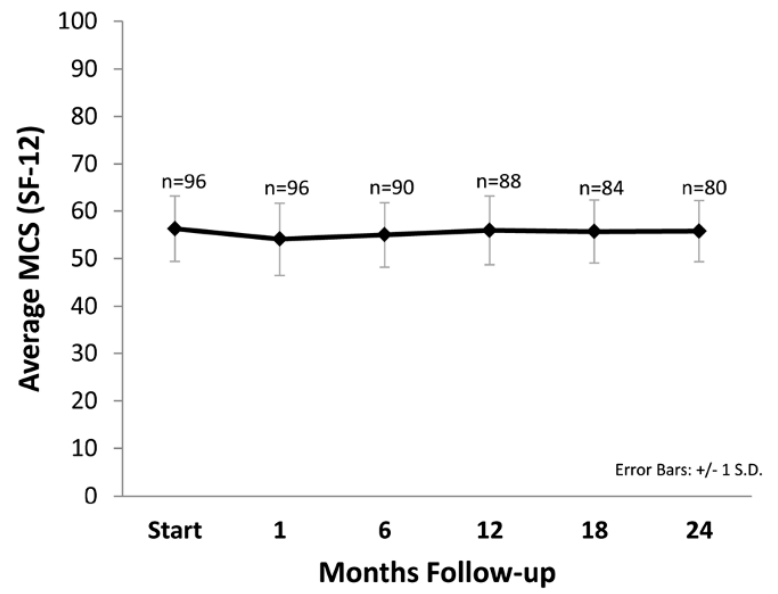

Figure 4 Short Form-12 (SF-12) Health Survey quality of life: (a) SF-12 physical component score (PCS) and (b) SF-12 mental component score (MCS). The graphs show unadjusted changes in average scores over time. The scores range from 0-100 with higher values representing improved health status. Numbers above each time point indicate the number of observations contributing to the average.

was 3 months (range, 3-9 months). Twenty-eight percent of the flares lasted longer than six months.

Prior to treatment, a significant portion of patients had erectile dysfunction based upon the SHIM (Table 4). We limited our sexual function analysis to the $57 \mathrm{pa}-$ tients who had a pretreatment SHIM score $\geq 10$ and who did not receive ADT. At two years post-treatment, the median SHIM decreased from a baseline of 19 to 18 $(p=0.003$, Figure $6 \mathrm{a})$. There was no statistically significant change in PDE5 inhibitor utilization over time (Figure $6 \mathrm{~b}$ ). At 2 -years post-treatment, $51 \%$ of patients had utilized a PDE5 inhibitor at some point during follow up. Seventy-nine percent of these patients maintained potency (as defined as a SHIM of $\geq 10$ ) at two year post-treatment (Table 5). The decline in 
Table 4 Pre-treatment Quality of Life (QOL) scores

\begin{tabular}{lcc}
\hline Baseline SF-12 Score & Pretreatment Score & \\
PCS $^{*}(n=96)$ & $48.9(19.7-60.8)$ & {$[S D=8.56]$} \\
$M C S^{*}(n=96)$ & $54.5(26.2-63.9)$ & {$[S D=6.88]$} \\
Baseline AUA Score & \% Patients $(n=100)$ & \\
0-7 (mild) & 46 \\
8-19 (moderate) & 47 \\
$\geq 20$ (severe) & 7 \\
Baseline SHIM Score & \% Patients $(n=98)$ \\
$<8$ (Severe ED) & 37 \\
8-11 (Moderate ED) & 2 \\
$12-16$ (Mild-Moderate ED) & 16 \\
17-21 (Mild ED) & 27 \\
22-25 (no ED) & 18 \\
\hline
\end{tabular}

${ }^{*}$ Norm-based scoring for SF-12 with population mean $=50$ and $S D=10$. Abbreviations: SF-12, Short-form-12; PCS, physical component score; MCS, mental component score; $S D$, standard deviation; $A U A$, American urological association; SHIM, sexual health inventory for men; $E D$, erectile dysfunction. potency at two years was unlikely due to aging, as the average age of potent patients was not statistically different from non-potent patients $(p=0.41)$.

\section{Discussion}

While IMRT and brachytherapy are the most commonly used radiation therapy modalities for clinically localized prostate cancer, SBRT utilization is increasing [19]. The accuracy assured by intra-fraction image guidance, which allows the use of smaller CTV-PTV margins, may allow safe prostate treatment in four or five large (e.g., $7-9.5 \mathrm{~Gy}$ ) radiation fractions. Emerging data from single institutional series [6-8] and a small multi-institutional Phase I study [20] suggest that this approach may provide similar clinical outcomes as other radiation modalities with high rates of biochemical control and low rates of grade 3 and higher toxicities. A recent update of grouped series confirmed SBRT achieved 5-year biochemical disease-free survival of $93 \%$ in patients with favorable prognosis [21]. Based on these reports, as well as patient preference for a shorter treatment course, SBRT utilization is likely to continue to increase.
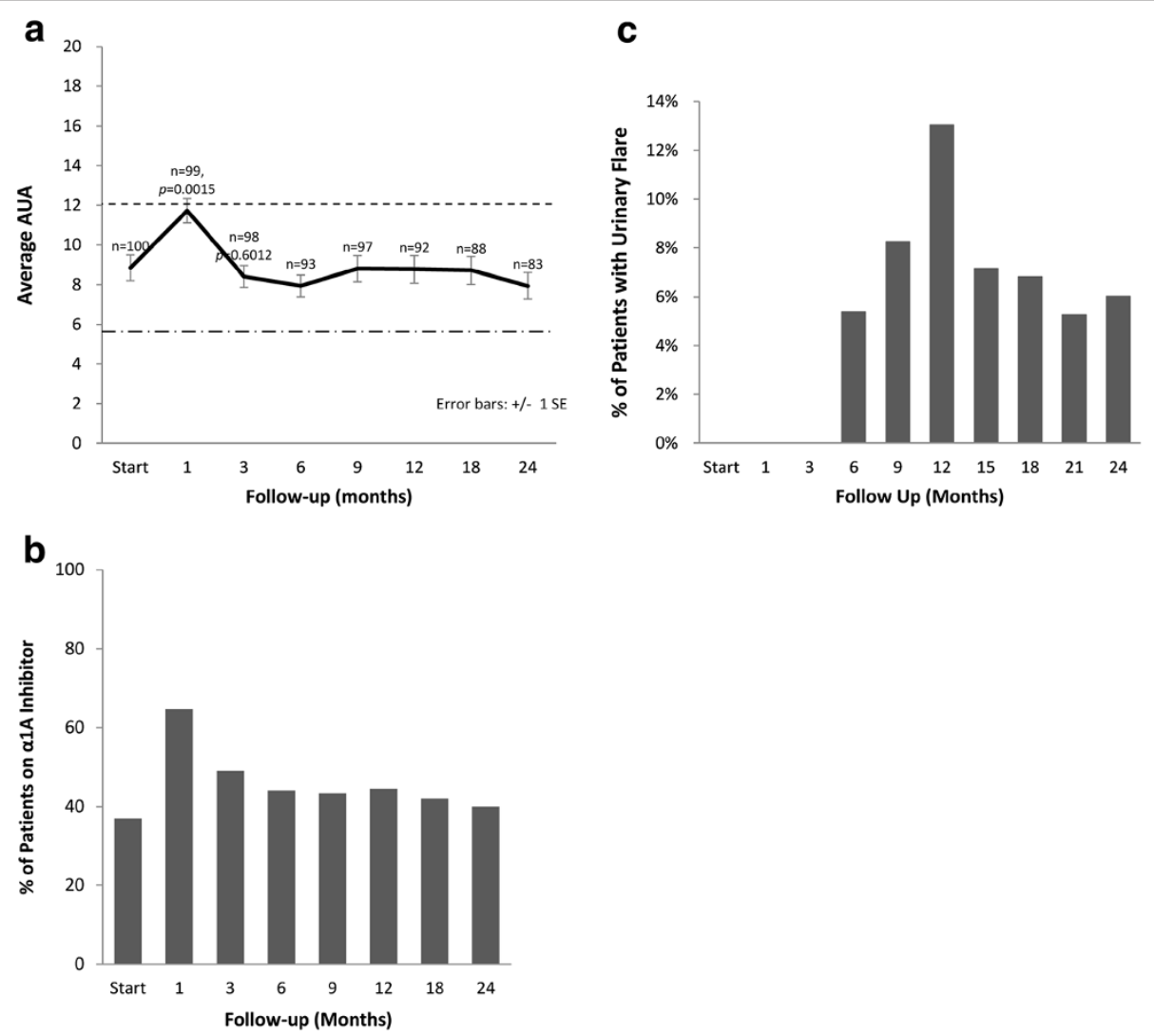

Figure 5 Urinary quality of life: (a) AUA score, (b) alpha antagonist utilization and (c) urinary symptom flare. The graphs show unadjusted changes in average scores over time for each domain. AUA scores range from 0-35 with higher values representing worsening urinary symptoms. Numbers above each time point indicate the number of observations contributing to the average. The thresholds for clinically significant changes in scores (1/2 standard deviation above and below the baseline) are marked with dashed lines. Error bars indicate $95 \%$ confidence intervals. 


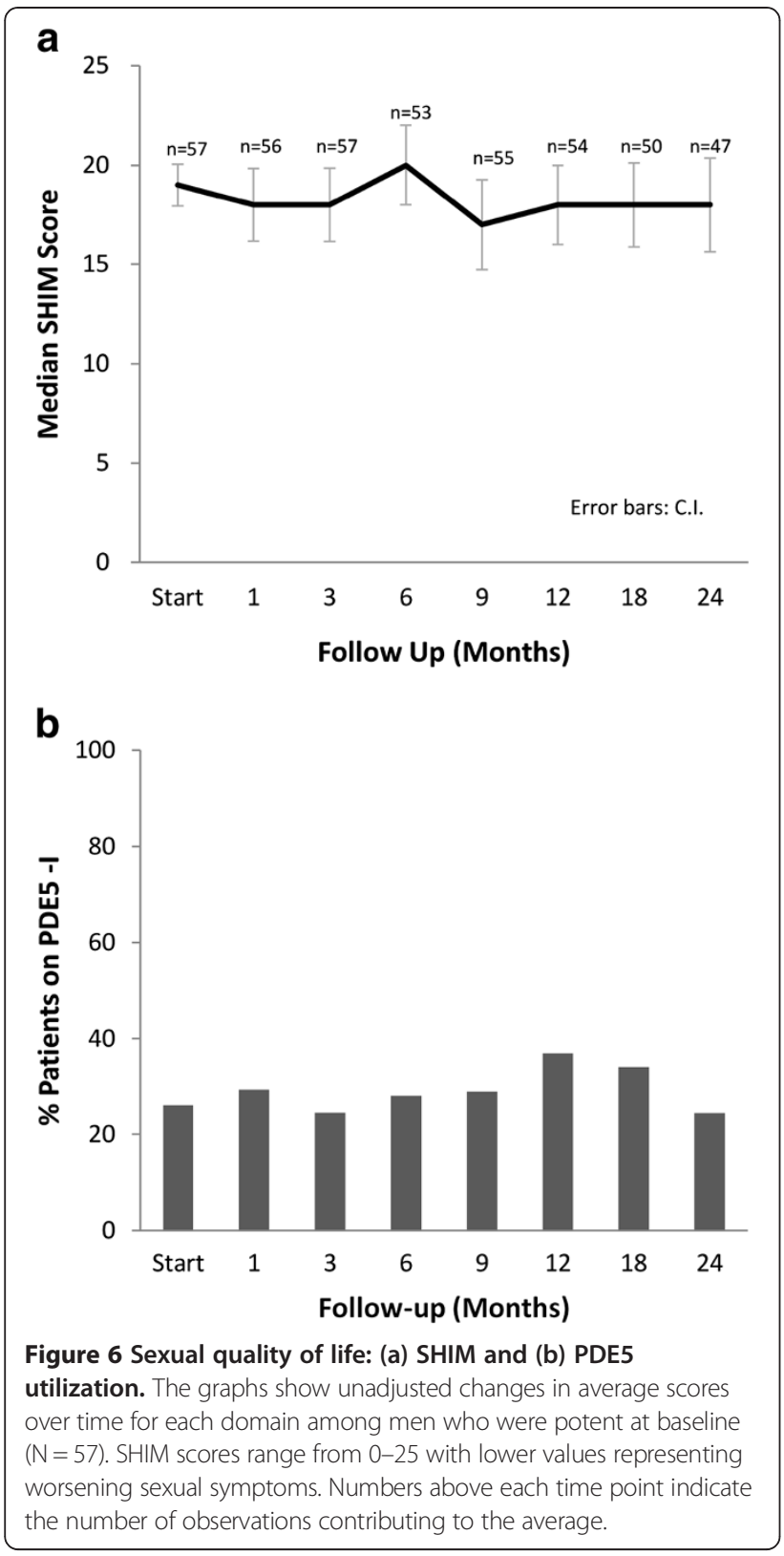

Our institutional experience adds to the growing body of evidence supporting the effectiveness and safety of SBRT. Our early PSA outcomes have been favorable. The two-year post-treatment PSA nadir of $0.49 \mathrm{ng} / \mathrm{mL}$ predicts a high rate of long-term disease control [22]. This PSA response is unlikely due to declines in testosterone since the majority of patients had stable testosterone over time. As with other SBRT series $[6,7,20]$ and other radiation therapy modalities [23,24], benign PSA bounces were common and transient. Considering that our series includes a higher percentage of intermediateand high-risk patients than others $[6-8,20]$, our $99 \%$ actuarial 2-year biochemical failure-free survival rate is reassuring.

There is limited data on the use of SBRT for unfavorable patients (6). To date, SBRT studies have included mostly favorable patients due to the concern over limited coverage of potential extracapsular extension and seminal vesicle invasion. A recent dosimetric study suggest that SBRT delivers adequate dose to areas of potential extracapsular extension and the proximal seminal vesicles in unfavorable patients [25]. Although prospective studies are needed to confirm long-term tumor control, currently available data are comparable to results reported for brachytherapy and conventional external beam radiotherapy $[26]$.

Toxicity following SBRT was similar to that following external beam radiation therapy or brachytherapy [27]. Late Grade 2 and Grade 3 GU toxicity were observed in $30 \%$ and $1 \%$ of patients, respectively (Figure 3 ). Alpha antagonist utilization was the most common grade 2 toxicity, but similar to brachytherapy treatment, it returned to baseline one to two years post-treatment [28]. To maximize patient comfort, it is currently our institutional policy to initiate prophylactic alpha antagonist use prior to initiating treatment. The single grade 3 toxicity was hematuria requiring a TURP. This patient had a history of benign prostatic hypertrophy with a large prostate and two prior TURP procedures and was dependent on intermittent catheterization prior to receiving SBRT. As others have, we recommend that urethral instrumentation following treatment should be

Table 5 SHIM scores at baseline and following treatment

\begin{tabular}{|c|c|c|c|c|c|c|c|c|}
\hline (Total N) & $\begin{array}{l}\text { Pre-Tx } \\
(57)\end{array}$ & $\begin{array}{c}1 \text { Mon } \\
(56)\end{array}$ & $\begin{array}{l}3 \text { Mon } \\
(57)\end{array}$ & $\begin{array}{c}6 \text { Mon } \\
(53)\end{array}$ & $\begin{array}{l}9 \text { Mon } \\
(55)\end{array}$ & $\begin{array}{c}12 \text { Mon } \\
(54)\end{array}$ & $\begin{array}{c}18 \text { Mon } \\
(50)\end{array}$ & $\begin{array}{c}24 \text { Mon } \\
(47)\end{array}$ \\
\hline$\%$ Potent $(\mathrm{SHIM} \geq 10)$ & $100.0 \%$ & $85.7 \%$ & $78.9 \%$ & $83.0 \%$ & $67.3 \%$ & $77.8 \%$ & $76.0 \%$ & $78.7 \%$ \\
\hline SHIM $22 \sim 25$ (No ED) & $28.1 \%$ & $26.0 \%$ & $22.2 \%$ & $28.2 \%$ & $22.0 \%$ & $24.5 \%$ & $22.8 \%$ & $28.5 \%$ \\
\hline SHIM 17 21 (Mild ED) & $43.9 \%$ & $27.6 \%$ & $24.9 \%$ & $25.1 \%$ & $15.9 \%$ & $20.2 \%$ & $16.7 \%$ & $16.7 \%$ \\
\hline SHIM 12 16 (Mild-Moderate ED) & $24.6 \%$ & $12.2 \%$ & $15.2 \%$ & $12.5 \%$ & $4.9 \%$ & $10.1 \%$ & $12.2 \%$ & $8.4 \%$ \\
\hline SHIM $8 \sim 11$ (Moderate ED) & $3.5 \%$ & $9.2 \%$ & $2.8 \%$ & $6.3 \%$ & $6.1 \%$ & $7.2 \%$ & $7.6 \%$ & $8.4 \%$ \\
\hline SHIM $<\mathbf{8}$ (Severe ED) & $0.0 \%$ & $10.7 \%$ & $13.9 \%$ & $11.0 \%$ & $18.3 \%$ & $15.8 \%$ & $16.7 \%$ & $16.7 \%$ \\
\hline
\end{tabular}

Abbreviations: Pre-Tx, pre-treatment; SHIM, sexual health inventory for men; $E D$, erectile dysfunction. 
limited in patients treated with SBRT [7]. The SF-12 scores showed no significant change throughout the follow-up period suggesting that toxicities did not significantly adversely affect the patients' perceptions of their health.

As seen by other SBRT series [20], our mean AUA scores returned to baseline by three months post treatment. However, a minority of patients experienced a clinically meaningful increase in their urinary symptoms greater than six months after the completion of treatment. To our knowledge this is the first reported series to characterize late urinary symptom flare $[17,29-32]$ in patients receiving SBRT. Like conventionally fractionated external beam radiation therapy and brachytherapy, late urinary toxicity occurred in a minority of our patients and resolved with conservative management (urinary anesthetics, alpha-blockers and/or brief steroid tapers). Endoscopic evaluation of these patients has suggested that this may be caused by urethritis (unpublished data). Knowledge of these late urinary toxicities and their resolution with conservative management will enable clinicians to relieve patient concerns and prevent unnecessary invasive procedures such as cystoscopy and/or TURP.

In our opinion, late urinary symptom flare may be exacerbated by the high central doses in our relatively inhomogeneous plans (Figure 1a). With the aim of reducing urinary symptoms, we have modified our institutional protocol. Specifically, we have reduced the anterior/superior PTV expansion to $3 \mathrm{~mm}$ to reduce the bladder neck dose. In addition, it is now our practice to prescribe to $\geq 80 \%$ isodose line to reduce the dose delivered to the prostatic urethra. Finally, to further reduce the prostatic urethral dose, we have restricted the maximum prostatic urethral dose to $110 \%$ of the prescription dose. We believe that such modifications will reduce the incidence and severity of late urinary symptom flare without increasing the risk of local failures $[9,10]$.

As in other radiation therapy series, our patients were elderly with poor baseline erectile function and high PDE5 inhibitor utilization prior to treatment [33-35]. Similar to other SBRT series $[8,36], 79 \%$ of patients who were sexually potent prior to treatment maintained potency at two years' post-treatment. These results are comparable to results with conventionally fractionated EBRT or brachytherapy [37,38]. Interestingly, as observed by others, the greatest decline in SHIM occurred in the first year with sexual function stabilization afterwards [39]. The etiology of this early decline in SHIM is uncertain. Fifty-one percent of patients utilized PDE5 inhibitors in follow-up. It is not clear why utilization was not higher following treatment. Potential explanations include patient indifference [33], limited effectiveness [40] or the high cost of such medications.
Our study should be examined in the context of the study design. Our study is limited by the retrospective nature of the analysis. However, subjects were accrued consecutively and all data were collected prospectively in a centralized database, therefore limiting selection and reporting bias present in pure retrospective studies. In addition, the narrow focus of the AUA on obstructive symptoms and SHIM on erectile function is another limitation of our study [41]. Future studies should employ more comprehensive instruments to assess the effect of prostate SBRT on overall urinary and sexual function in this elderly patient population.

\section{Conclusions}

SBRT was well-tolerated for these patients with clinically localized prostate cancer. Early PSA results suggest a biochemical response similar to other standard radiation therapy options. Benign PSA bounces were common. Rates of late GI and GU toxicity are comparable to conventionally fractionated radiation therapy and brachytherapy. Late urinary symptom flares are observed but the majority resolved with conservative management. A high percentage of men who were potent prior to treatment remained potent two years following treatment.

\section{Abbreviations \\ ADT: Androgen deprivation therapy; AUA: American urological association; CTC: Common toxicity criteria; CTV: Clinical target volume; DVH: Dose- volume histogram; Gl: Gastrointestinal; GU: Genitourinary; GTV: Gross target volume; PTV: Planning target volume; QoL: Quality of life; SHIM: Sexual health inventory for men; SBRT: Stereotactic body radiation therapy; SF-12: Short Form-12 health survey.}

\section{Competing interests}

SP Collins and BT Collins serve as clinical consultants to Accuray Inc. The Department of Radiation Medicine at Georgetown University Hospital receives a grant from Accuray to support a research coordinator. The other authors declare that they have no competing interests.

\section{Authors' contributions}

LC and SS are lead authors, who participated in data collection, data analysis, manuscript drafting, table/figure creation and manuscript revision. SU aided in the quality of life data collection and maintained the patient database. EO aided in the quality of life data collection and statistical analysis. AJ aided in the quality of life data collection. VC aided in the quality of life data collection. $\mathrm{HH}$ aided in the quality of life data collection and maintained the patient database. SL aided in the quality of life data collection. JK aided in the quality of life data collection and maintained the patient database, aided in data collection, and participated in initial data interpretation. SL is the dosimetrist who developed the majority of patients' treatment plans, and contributed to the dosimetric data analysis and interpretation. GP, KK, GB, JP, KM, aided in clinical data collection. BC, PK, ND, participated in the design and coordination of the study. KT aided in statistical analysis, quality of life analysis and manuscript revision. $A D$ is a senior author who aided in drafting the manuscript. $J$ is a senior author who aided in drafting the manuscript. SC was the principal investigator who initially developed the concept of the study and the design, aided in data collection, drafted and revised the manuscript. All authors read and approved the final manuscript.

\section{Acknowledgements}

We acknowledge Daniel Hamstra, M.D., Ph.D., Robert Meier, M.D., Debra Freeman, M.D., Alan Katz, M.D. and Donald Fuller, M.D. for helpful discussions. 


\section{Author details}

'Department of Radiation Medicine, Georgetown University Hospital, Washington, DC 20007, USA. ²Department of Urology, Georgetown University Hospital, Washington, DC 20007, USA. ${ }^{3}$ Department of Radiology, Georgetown University Hospital, Washington, DC 20007, USA. ${ }^{4}$ Department of Oncology, Lombardi Comprehensive Cancer Center, Georgetown University Medical Center, Washington, DC 20007, USA.

Received: 25 October 2012 Accepted: 25 February 2013

Published: 13 March 2013

\section{References}

1. Fowler JF: The radiobiology of prostate cancer including new aspects of fractionated radiotherapy. Acta Oncol 2005, 44:265-276.

2. Miles EF, Lee WR: Hypofractionation for prostate cancer: a critical review. Semin Radiat Oncol 2008, 18:41-47.

3. Madsen BL, Hsi RA, Pham HT, Fowler JF, Esagui L, Corman J: Stereotactic hypofractionated accurate radiotherapy of the prostate (SHARP), 33.5 Gy in five fractions for localized disease: first clinical trial results. Int J Radiat Oncol Biol Phys 2007, 67:1099-1105.

4. Hossain S, Xia P, Huang K, et al: Dose gradient near target-normal structure interface for nonisocentric CyberKnife and isocentric intensitymodulated body radiotherapy for prostate cancer. Int J Radiat Oncol Biol Phys 2010, 78:58-63.

5. Xie Y, Djajaputra D, King CR, Hossain S, Ma L, Xing L: Intrafractional motion of the prostate during hypofractionated radiotherapy. Int J Radiat Oncol Biol Phys 2008, 72:236-246.

6. Katz AJ, Santoro M, Ashley R, Diblasio F, Witten M: Stereotactic body radiotherapy for organ-confined prostate cancer. BMC Urol 2010, 10:1.

7. King CR, Brooks JD, Gill H, Presti JC Jr: Long-term outcomes from a prospective trial of stereotactic body radiotherapy for low-risk prostate cancer. Int J Radiat Oncol Biol Phys 2011, 82:877-882.

8. Friedland JL, Freeman DE, Masterson-McGary ME, Spellberg DM: Stereotactic body radiotherapy: an emerging treatment approach for localized prostate cancer. Technol Cancer Res Treat 2009, 8:387-392.

9. Vainshtein J, Abu-lsa E, Olson KB, et al: Randomized phase II trial of urethral sparing intensity modulated radiation therapy in low-risk prostate cancer: implications for focal therapy. Radiat Oncol 2012, 7:82

10. Nguyen $\mathrm{PL}$, Chen $\mathrm{MH}$, Zhang $\mathrm{Y}$, et al: Updated Results of Magnetic Resonance Imaging Guided Partial Prostate Brachytherapy for Favorable Risk Prostate Cancer: Implications for Focal Therapy. J Urol 2012, 188:1151-1156.

11. Ware J Jr, Kosinski M, Keller SD: A 12-Item Short-Form Health Survey: construction of scales and preliminary tests of reliability and validity. Med Care 1996, 34:220-233.

12. Barry MJ, Fowler FJ Jr, O'Leary MP, et al: The American Urological Association symptom index for benign prostatic hyperplasia. The Measurement Committee of the American Urological Association. J Uro 1992, 148:1549-1557. discussion 1564

13. Rosen RC, Cappelleri JC, Gendrano N 3rd: The International Index of Erectile Function (IIEF): a state-of-the-science review. Int J Impot Res 2002, 14:226-244.

14. Ciezki JP, Reddy CA, Garcia J, et al: PSA kinetics after prostate brachytherapy: PSA bounce phenomenon and its implications for PSA doubling time. Int J Radiat Oncol Biol Phys 2006, 64:512-517.

15. Ware JE Jr, Kosinski M, Bayliss MS, McHorney CA, Rogers WH Raczek A: Comparison of methods for the scoring and statistical analysis of SF-36 health profile and summary measures: summary of results from the Medical Outcomes Study. Med Care 1995, 33:AS264-279.

16. Norman GR, Sloan JA, Wyrwich KW: Interpretation of changes in healthrelated quality of life: the remarkable universality of half a standard deviation. Med Care 2003, 41:582-592

17. Crook J, Fleshner N, Roberts C, Pond G: Long-term urinary sequelae following 125iodine prostate brachytherapy. J Uro/ 2008, 179:141-145. discussion 146.

18. Brown MW, Brooks JP, Albert PS, Poggi MM: An analysis of erectile function after intensity modulated radiation therapy for localized prostate carcinoma. Prostate Cancer Prostatic Dis 2007, 10:189-193.

19. Pan H, Simpson DR, Mell LK, Mundt AJ, Lawson JD: A survey of stereotactic body radiotherapy use in the United States. Cancer 2011, 117:4566-4572.
20. McBride SM, Wong DS, Dombrowski JJ, et al: Hypofractionated stereotactic body radiotherapy in low-risk prostate adenocarcinoma: Preliminary results of a multi-institutional phase 1 feasibility trial. Cancer 2012, 118:3681-3690.

21. Freeman $D E$, King CR: Stereotactic body radiotherapy for low-risk prostate cancer: five-year outcomes. Radiat Oncol 2011, 6:3.

22. Zelefsky MJ, Shi W, Yamada Y, et al: Postradiotherapy 2-year prostatespecific antigen nadir as a predictor of long-term prostate cancer mortality. Int J Radiat Oncol Biol Phys 2009, 75:1350-1356.

23. Crook J, Gillan C, Yeung I, Austen L, McLean M, Lockwood G: PSA kinetics and PSA bounce following permanent seed prostate brachytherapy. Int J Radiat Oncol Biol Phys 2007, 69:426-433.

24. Horwitz EM, Levy LB, Thames HD, et al: Biochemical and clinical significance of the posttreatment prostate-specific antigen bounce for prostate cancer patients treated with external beam radiation therapy alone: a multiinstitutional pooled analysis. Cancer 2006, 107:1496-1502.

25. Ju AW, Suy S, Lei S, et al: Hypofractionated stereotactic body radiation therapy as monotherapy for intermediate-risk prostate cancer. Radiat Oncol. In Press.

26. Katz AJ: Stereotactic body radiotherapy for prostate cancer: ready for prime time? J Radiat Oncol 2012, 1:17-30.

27. Tsui G, Gillan C, Pond G, Catton C, Crook J: Posttreatment complications of early-stage prostate cancer patients: brachytherapy versus threedimensional conformal radiation therapy. Cancer J 2005, 11:122-132.

28. Merrick GS, Butler WM, Lief JH, Dorsey AT: Temporal resolution of urinary morbidity following prostate brachytherapy. Int J Radiat Oncol Biol Phys 2000, 47:121-128.

29. Schmid MP, Potter $R$, Bombosch $V$, et al: Late gastrointestinal and urogenital side-effects after radiotherapy-incidence and prevalence. Subgroup-analysis within the prospective Austrian-German phase II multicenter trial for localized prostate cancer. Radiother Oncol 2012, 104:114-118.

30. Fonteyne V, Villeirs G, De Lumen N, Meerleer G: Urinary toxicity after high dose intensity modulated radiotherapy as primary therapy for prostate cancer. Radiother Oncol 2009, 92:42-47.

31. Keyes M, Miller S, Moravan V, et al: Urinary symptom flare in 712125 prostate brachytherapy patients: long-term follow-up. Int J Radiat Oncol Biol Phys 2009, 75:649-655.

32. Cesaretti JA, Stone NN, Stock RG: Urinary symptom flare following I-125 prostate brachytherapy. Int J Radiat Oncol Biol Phys 2003, 56:1085-1092.

33. Miller DC, Wei JT, Dunn RL, et al: Use of medications or devices for erectile dysfunction among long-term prostate cancer treatment survivors: potential influence of sexual motivation and/or indifference. Urology 2006, 68:166-171.

34. Bergman J, Gore JL, Penson DF, Kwan L, Litwin MS: Erectile aid use by men treated for localized prostate cancer. J Urol 2009, 182:649-654.

35. Stephenson RA, Mori M, Hsieh YC, et al: Treatment of erectile dysfunction following therapy for clinically localized prostate cancer: patient reported use and outcomes from the Surveillance, Epidemiology, and End Results Prostate Cancer Outcomes Study. J Urol 2005, 174:646-650. discussion 650

36. Wiegner EA, King CR: Sexual function after stereotactic body radiotherapy for prostate cancer: results of a prospective clinical trial. Int J Radiat Oncol Biol Phys 2010, 78:442-448.

37. Merrick GS, Butler WM, Wallner KE, et al: Erectile function after prostate brachytherapy. Int J Radiat Oncol Biol Phys 2005, 62:437-447.

38. Sanda MG, Dunn RL, Michalski J, et al: Quality of life and satisfaction with outcome among prostate-cancer survivors. N Engl J Med 2008, 358:1250-1261.

39. Siglin J, Kubicek GJ, Leiby B, Valicenti RK: Time of decline in sexual function after external beam radiotherapy for prostate cancer. Int $J$ Radiat Oncol Biol Phys 2010, 76:31-35.

40. Teloken PE, Parker M, Mohideen N, Mulhall JP: Predictors of response to sildenafil citrate following radiation therapy for prostate cancer. J Sex Med 2009, 6:1135-1140.

41. Levinson AW, Ward NT, Sanda MG, et al: Comparison of validated instruments measuring sexual function in men. Urology 2010, 76:380-386.

doi:10.1186/1748-717X-8-58

Cite this article as: Chen et al:: Stereotactic Body Radiation Therapy (SBRT) for clinically localized prostate cancer: the Georgetown University experience. Radiation Oncology 2013 8:58. 